\title{
Effects of Color Temperature and Brightness on Electroencephalogram Alpha Activity in a Polychromatic Light-emitting Diode
}

\author{
Jin Young Park ${ }^{1,2}$, Ra-Yeon $\mathrm{Ha}^{1,2}$, Vin Ryu ${ }^{3}$, Eosu Kim ${ }^{1,2}$, Young-Chul Jung ${ }^{1,2}$ \\ ${ }^{1}$ Department of Psychiatry, ${ }^{2}$ Institute of Behavioral Science in Medicine, Yonsei University College of Medicine, Seoul, ${ }^{3}$ Department of \\ Psychiatry, Konyang University College of Medicine, Daejeon, Korea
}

\begin{abstract}
Objective: It has been reported that working and learning efficiency might be increased through artificially controlling the color temperature and brightness of light. However, the neurological bases of these outcomes are not well established. Our study was designed to observe whether electroencephalogram (EEG) alpha frequency, as a candidate biological marker, demonstrates significant changes in response to alterations of specific light parameters.

Methods: Thirty-two healthy subjects performed cognitive tasks under four different polychromatic light conditions: a combination of two different levels of color temperature (2766K vs. 5918K) and brightness (300 lux vs. 600 lux). Spectrum analyses were performed on alpha frequency.

Results: Subjects reported that they felt more pleasant in bright conditions and more relaxed in warm color temperature conditions. Our findings indicate that alpha power increases in warm, low-light and cool, high-light conditions, and there is a significant interaction between color temperature and brightness.

Conclusion: EEGs might serve as a useful biological marker for further research related to the effects of polychromatic light on cognitive function.
\end{abstract}

KEY WORDS: Polychromatic light; Color temperature; Brightness; Electroencephalogram; Alpha frequency.

\section{INTRODUCTION}

Light has been traditionally considered an important element related to the visual pathway. However, the discovery of melanopsin, a photopigment in specialized, photosensitive ganglion cells of the retina, has elicited great attention to the effects of light through the non-visual pathway, which is involved in the regulation of circadian rhythm, light-dark cycle phase shifts, pupil constriction, melatonin secretion, and homeostatic sleep drive control. $^{1,2)}$ Recently, it has been proposed that light may have direct effects on cognitive function, such as attention, executive function, and memory. ${ }^{3)}$

Most experimental studies investigating the effects of light have primarily used monochromatic light, which is a short-wavelength light representing only one color. How-

\footnotetext{
Received: November 26, 2012 / Revised: February 27, 2013 Accepted: March 7, 2013

Address for correspondence: Young-Chul Jung, MD, PhD Department of Psychiatry, Severance Mental Health Hospital,Yonsei University College of Medicine, 696-6 Tanbeol-dong, Gwangju 464-100, Korea

Tel: +82-31-760-9501, Fax: +82-31-760-950

E-mail: eugenejung@yuhs.ac
}

ever, in everyday life, most indoor lights are polychromatic light, which is the sum of various frequency waves that express a diverse spectrum of brightness and color temperature. The color of polychromatic light is red-enriched white, usually referred to as 'warm white,' when the numerical value of color temperature is less than $3500 \mathrm{~K}$. However, it changes to blue-enriched white, usually referred as 'cool white,' when the color temperature increases and reaches $10000 \mathrm{~K}$. Low-color temperature lighting can be found in light bulbs that emanate a serene and stable mood. In contrast, high-color temperature lighting, such as fluorescent lights, creates a more active atmosphere. ${ }^{4}$ The wellbeing and productivity of employees in workplaces are known to increase under light conditions of high color temperature $(17000 \mathrm{~K}){ }^{5)}$ In addition, providing an environment of ample bright light (1000 lux) was reported to delay the progression of cognitive deterioration and functional limitation among elderly residents and attenuate their symptoms of depression. ${ }^{6}$ Based on these reports, it has been suggested that controlling color temperature and brightness is critical to create an appropriate indoor working environment ${ }^{7)}$ and improve work

(a) This is an Open-Access article distributed under the terms of the Creative Commons Attribution Non-Commercial License (http://creativecommons.org/licenses/by-nc/3.0) which permits unrestricted non-commercial use, distribution, and reproduction in any medium, provided the original work is properly cited. 
performance. ${ }^{8)}$

Although a number of studies investigated the direct effects of polychromatic light on cognitive function and mood, most relied on self-reported questionnaires with little information on the neural mechanisms of these effects. In the 1990s, a few studies applied electroencephalogram (EEG) analyses, ${ }^{9,10)}$ but it was difficult to investigate the effects of polychromatic light, as controlling color temperature and brightness was limited. Recently, the development of light-emitting diodes (LEDs) has enabled more standardized control of light conditions, including color temperature and brightness. ${ }^{11)}$ Therefore, using an LED as a light source makes it more feasible to study the biological markers impacted by various levels of color temperature and brightness.

Alpha oscillations of a normal human EEG has a frequency range of 8 to $13 \mathrm{~Hz}$. Alpha oscillations respond very sensitively to external sensory input. For example, closing one's eyes (that is, decreasing visual sensory input to the occipital areas) leads to an increase of the power of occipital alpha oscillations. ${ }^{12)}$ On the other hand, alpha oscillations are proposed to be associated with neuronal mechanisms of complex cognitive processes, including attention, memory, and mental imagery. ${ }^{13)}$ In addition, according to studies of monochromatic light, alpha activity is sensitive to blue and red monochromatic lights. ${ }^{14)}$ It was also found that alpha power density increased in response to short-wavelength light. ${ }^{3)}$ Thus, the frequency of alpha oscillations can be a feasible biological marker to observe the effects of external light conditions on cognition.

This study investigated the direct effects of polychromatic light on EEG activity. We hypothesized that alpha band activity would reflect the differences in environmental light conditions during cognitive tasks. To confirm this hypothesis, a cognitive task was executed that measured the alpha activity under four different light conditions: a combination of different levels of color temperature $(2766 \mathrm{~K}$ vs. $5918 \mathrm{~K})$ and brightness (300 lux vs. 600 lux), ranges that are commonly used in indoor environments.

\section{METHODS}

Thirty-two subjects participated in this study (16 females; age, $26.81 \pm 4.17$ years; education, $15.56 \pm 1.44$ years). All participants were screened for past or present history of medical, neurological, and psychiatric illnesses. Persons who worked the nightshift or who usually went to bed after 1:00 A.M. were excluded from the study. Sub- jects taking any kind of prescribed medication were also excluded from the study, and we excluded those who had color blindness, color weakness, and other ophthalmic disorders. The subjects, on average, consumed alcohol 0.39 times (standard deviation [SD] 0.42) per week, 2.95 glasses (SD 3.69) each time. Only five participants were smokers, and they smoked 0.5-1.5 packs/year. The participants were instructed to sleep regularly and to abstain from alcohol for at least 3 days. The participants were prohibited from caffeine or nicotine for at least 2 hours proceeding the session. After a complete description of the study was provided to the participants, written informed consent was obtained. Out of 32 subjects in total, 16 began the test at 10:00 A.M. and 16 began the test at 3:00 P.M. Our study was carried out under the guidelines for the use of human subjects established by the Institutional Review Board at Severance Mental Health Hospital, Yonsei University.

Experiments were conducted under four different conditions according to color temperature and brightness: warm, low-light (WL, 2766K 300 lux); cool, low-light (CL, 5918K 300 lux); warm, high-light (WH, 2766K 600 lux); and cool, high-light (CH, 5918K 600 lux). The photon density of each condition was as follows: WL was $1.61 \times 10^{13}$ (photons $/ \mathrm{cm}^{2} / \mathrm{s}$ ); CL was $9.92 \times 10^{12}$ (photons/ $\mathrm{cm}^{2} / \mathrm{s}$ ); WH was $3.48 \times 10^{13}$ (photons $/ \mathrm{cm}^{2} / \mathrm{s}$ ); and $\mathrm{CH}$ was $3.07 \times 10^{13}$ (photons $/ \mathrm{cm}^{2} / \mathrm{s}$ ). Sources of light $(54 \times 54 \mathrm{~cm}$; LG Electronics, Seoul, Korea) were suspended from the ceiling (207 $\mathrm{cm}$ from the ground), whereas color temperature and illuminance were measured from a desk with a height that measured $70 \mathrm{~cm}$ from ground level.

Each light condition consisted of three blocks. Subjects were first seated comfortably in a dark condition ( 0 lux) for 5 minutes (prepare block). Subjects were asked to perform a cognitive task in one of the light conditions for 5 minutes (concentration block). After the cognitive task, the participants were asked to be seated in a relaxed state for 5 minutes (relaxation block; Fig. 1). Each light condition required 15 minutes, and the order of the four different light conditions was counter-balanced. During the concentration block, questions from Raven's Standard Progressive Matrices and Raven's Advanced Progressive Matrices were randomly presented to the subjects through an e-book (Iriver, Seoul, Korea), which itself had no luminous source to control for any light bias. Subjects were asked to solve questions quickly and accurately. During the relaxation block, subjects were asked to open their eyes and sit comfortably without performing any task. At the end of each light condition, the participants reported 
their subjective feelings on valence and arousal using a 11-point Likert scale. ${ }^{15)}$ The endpoints for the valence scale were anchored at very unhappy and very happy, whereas the endpoints for the arousal scale were anchored at agitated and relaxed.

During the experiment, EEG measurements were taken from the scalp using a SynAmps2 DC amplifier and a 10/20-layout, 64-channel, Quik-cap electrode placement system (Neuroscan Inc., Charlotte, NC, USA). We re- corded EEG data with a $0.15-200-\mathrm{Hz}$ band-pass filter at a sampling rate of $1,000 \mathrm{~Hz}$. The ground electrode was placed on the forehead. We used the linked mastoid for reference and two bipolar electrodes to measure the horizontal and vertical eye movements. The impedance of each electrode was maintained below $10 \mathrm{k} \Omega$. We used Matlab 7.0.1 (MathWorks, Natick, MA, USA) with the EEGLAB toolbox ${ }^{16)}$ to preprocess and analyze the data. First, the data were detrended and the means subtracted.

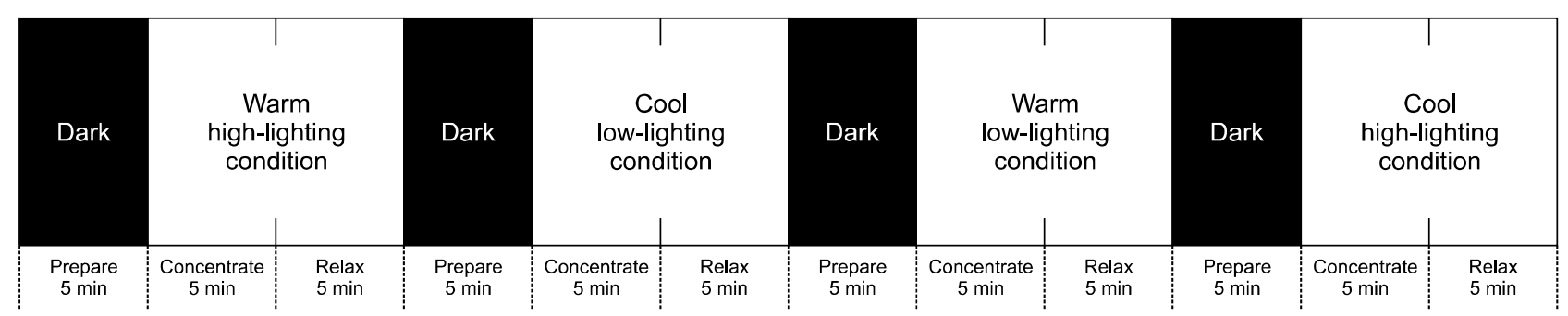

Fig. 1. Experimental design. The order of the four different light conditions was counter-balanced.

A

Very unpleasant

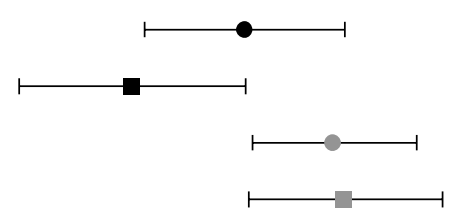

Very pleasant
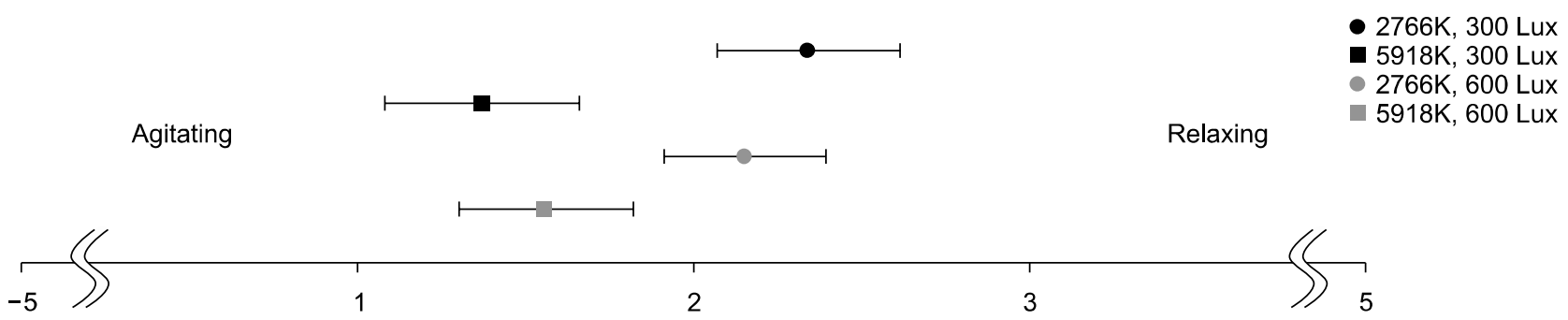

B

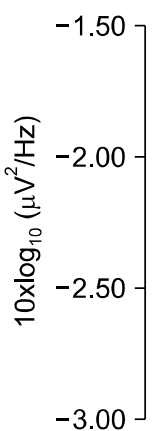

$\mathrm{Fz}$

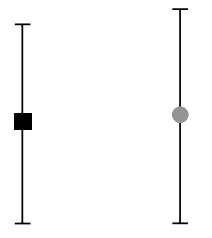

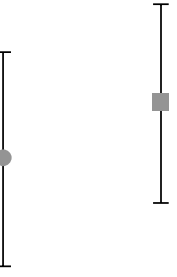

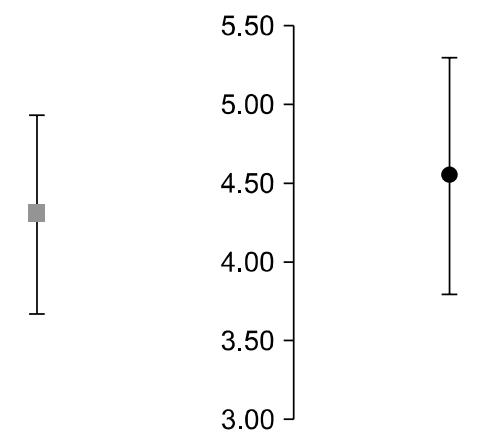

$\mathrm{Oz}$

Fig. 2. (A) The scores of subjective impression. (B) The power spectra of alpha rhythms in the Fz and Oz electrodes (error bars indicate the standard error). 
Independent component decomposition and visual inspection of the data were performed to eliminate artifacts. The average power of alpha $(8-13 \mathrm{~Hz})$ was calculated at both the $\mathrm{Fz}$ and $\mathrm{Oz}$ electrodes during the concentration condition. We selected the most representative Z-line channel, considering that the occipital area is most sensitive to visual stimulation and that the frontal area is crucial for cognitive processing. When the light was turned on, we analyzed EEG data acquired between 120 and 180 seconds. There was a possibility that the EEGs before and after the light changes were affected by external factors; according to a preliminary analysis of this experiment, it was possible to obtain stable EEG power when the signal lasted for 60 seconds. For this reason, the time range between 120 and 180 seconds was chosen. Alpha power for each electrode was analyzed by a repeated-measures analysis of variance (ANOVA), comprising color temperature and brightness as within-subject factors. Significant $p$ values were defined as less than 0.05 .

\section{RESULTS}

Subjects reported that they felt happier in brighter conditions and more relaxed in warmer color temperature conditions. Repeated-measures ANOVA indicated that there was a significant effect of brightness on the valence scores $[\mathrm{F}(1,31)=6.183, p=0.018]$. However, there was neither a significant effect of color temperature on valence scores $[\mathrm{F}(1,31)=0.395, p=0.534]$ nor a significant interaction between brightness and color temperature $[\mathrm{F}(1,31)$ $=1.601, p=0.215]$. Arousal scores varied significantly by color temperature $[\mathrm{F}(1,31)=17.566, p<0.001]$ but not by brightness $[\mathrm{F}(1,31)=0.000, p=1.000]$. Significant interactions between brightness and color temperature were not found in arousal scores $[\mathrm{F}(1,31)=1.251, p=0.272]$ (Fig. 2A).

Alpha activities were sensitive to light conditions. Re-

Table 1. Repeated-measures ANOVA on alpha power during the cognitive task

\begin{tabular}{cccc}
\hline & Variable & $F$ & $P$ value \\
\hline Fz & CT & 0.902 & 0.350 \\
& B & 0.855 & 0.362 \\
& CT $\times$ B & 9.141 & $0.005^{\star}$ \\
Oz & CT & 0.033 & 0.857 \\
& B & 4.437 & $0.045^{\dagger}$ \\
& CT $\times$ B & 8.375 & $0.007^{\star}$
\end{tabular}

${ }^{*} p<0.01,{ }^{\dagger} p<0.05$.

ANOVA, analysis of variance; CT, color temperature; $B$, brightness. peated-measures ANOVA showed significant interactions between color temperature and brightness at both the $\mathrm{Fz}$ and $\mathrm{Oz}$ electrodes (Table 1, Fig. 2B) [Fz: $\mathrm{F}(1,31)=9.141$, $p=0.005$; Oz: $\mathrm{F}(1,31)=8.375, p=0.007]$. As shown in Fig. $2 \mathrm{~B}$, alpha powers under WL and $\mathrm{CH}$ conditions were higher than those under WH and CL conditions. Additionally, at the $\mathrm{Oz}$ electrode, alpha power was lower in high-light conditions than in low-light conditions $[\mathrm{F}(1,31)=4,437$, $p=0.045]$.

\section{DISCUSSION}

The main finding of our research is that the interaction between color temperature and brightness affected alpha activity in the frontal and occipital areas. Based on the Kruithof curve, ${ }^{17)}$ it has been consistently suggested that both color temperature and brightness should be considered to find optimal light conditions for working and living environments. ${ }^{18)}$ It is worth noting that higher alpha powers were found in WL and $\mathrm{CH}$ conditions compared to the other two light conditions. WL and $\mathrm{CH}$ lights are widely used in everyday life in the form of light bulbs and fluorescent lamps. According to Kruithof, ${ }^{17)}$ the general experience of light as pleasant or unpleasant is determined by the relationship between color temperature and brightness. In general, light with a lower color temperature (e.g., $3000 \mathrm{~K}$ ) provides pleasing illumination between 100 to 300 lux or higher, whereas sources that are rated cooler should be used at a proportionately higher illuminance. The WL and $\mathrm{CH}$ conditions were empirically assessed as being pleasing, whereas WH and CL conditions were considered displeasing. ${ }^{17)}$ Our results on alpha activity may reflect this interactive effect between color temperature and brightness.

The increased alpha activity in the WL and $\mathrm{CH}$ conditions compared to the $\mathrm{WH}$ and $\mathrm{CL}$ conditions suggests the inhibition of non-task-relevant processes. Alpha oscillations are thought to be associated with neuronal mechanisms of complex cognitive processes, such as attention, memory, and mental imagery. ${ }^{13)}$ Alpha synchronization appears to be related to active inhibition, whereas alpha desynchronization is corrleated with release from inhibition. ${ }^{19)}$ Alpha oscillations are strengthened by internal tasks, ${ }^{20)}$ which reflect the inhibition of the non-task-relevant process. Standard Progressive Matrices ${ }^{21)}$ measure the function of formal reasoning, ${ }^{22)}$ and this cognitive process requires the integration of relationships, the ability to infer rules and form high-level abstractions, and the inhibition of salient distracters. ${ }^{23)}$ Taken together, in- 
hibitory processing might occur more strongly in WL and $\mathrm{CH}$ conditions during problem-solving, when the alpha powers are likely to be increased.

We were able to find differences in alpha power, depending on the lighting level only in the occipital area, but not in the frontal area. Closing the eyes, and therefore decreasing sensory input to the occipital-parietal areas, leads to the increase of the power of alpha oscillations, whereas opening the eyes attenuates it. ${ }^{24)}$ In general, this suggests that alpha powers are lower in high-light conditions than in low-light conditions, perhaps reflecting that alpha powers are enhanced when brightness is decreased. To confirm this hypothesis, we calculated the alpha powers during the relaxing conditions. If decreasing sensory input in a low luminance condition correlates with an increase in alpha power during the concentration condition, relaxing conditions would have the same effect. However, we did not find any significant effect of alpha power in the relaxing conditions (Supplementary Table 1). This study was a preliminary experiment, and future research is necessary to clarify this issue.

The subjective impression scales from this study showed high valence scores in brighter light conditions and low arousal scores in low-color temperature conditions. It was found that bright light ameliorates depressive symptoms among elderly subjects and improves subjective ratings of mood, energy, and productivity among people who have seasonal affective symptoms in the workplace. ${ }^{25)}$ In addition, lights with lower color temperature were found convey serenity and stability during the biologically relaxing period. ${ }^{18)}$ The results from this study were consistent with findings from previous studies $^{18,26)}$ in that the subjects' happiness increased in brighter light conditions and became more relaxed under lights with lower color temperature.

It has been suggested that valence and arousal affect EEGs and that these two affective elements are reflected in different components. For example, P3a showed enhanced amplitudes in response to unpleasant stimuli and $\mathrm{P} 3 \mathrm{~b}$ was sensitive to the arousal value of the stimulation. ${ }^{27)}$ Additionally, both valence and arousal are known to affect cognitive processes. ${ }^{28)}$ Previous studies have applied extreme values of color temperature and brightness that are rarely used in practice, such as $1000 \operatorname{lux}^{6)}$ or $17000 \mathrm{~K}^{5)}$ However, the color temperature and brightness of our lighting conditions were within the ranges that are commonly used in indoor working and living environments. This difference might explain the low statistical power of our findings.
Alpha rhythmic oscillations are generated in the interactions between thalamocortical neurons and $\gamma$-aminobutyric acid (GABA)ergic cells in the thalamic reticular nucleus. ${ }^{12)}$ Recent neuroimaging studies showed that the thalamus is activated in response to consistent light exposure during cognitive tasks and involved in the interaction between alertness and cognition during executive tasks. ${ }^{29)}$ The thalamus is called "the gateway to the cortex," because of its critical role in controlling the flow of information from receptors to the sensory areas of the cortex. Almost all sensory information enters the cortex through the thalamus. In addition to sensory-related functions, the thalamus also plays an important role in modulating the flow of information in the cognitive, motor, and affective cortical areas. ${ }^{12)}$ In this study, in which subjects were asked to perform cognitive tasks under different light conditions, results showed that the alpha power of the subjects significantly differed according to the specific light conditions. This suggest that the difference in light stimuli was reflected in the thalamus, which controls the processing of sensory-related stimuli and cognitive performance, resulting in differentially generated alpha oscillations from thalamocortical interactions.

In this study, we found that alpha activity reacts to conditional differences in polychromatic light when performing cognitive tasks. As mentioned earlier, many researchers have tried to improve quality of life by controlling the color temperature and brightness of light in work, school, and home environments. However, little is known about the processes that mediate the effects of these parameters of light.

This study identified that alpha power is an EEG signal that is sensitive to alterations in polychromatic light. This result somewhat suggests that alpha power is a candidate biological marker. Based on this study, we expect to elucidate the effects of light on humans in greater detail by further varying the conditions of color temperature and brightness, and assessing the performance of various cognitive tasks under resting conditions.

\section{- Acknowledgments}

This work was supported by a 2010 research grant from LG electronics. The authors thank Kyung Li Park for helping to write manuscript. We also thank to Se Joon You and Yeon Hong Jung (LG Electronics) for providing LED devices and controlling it. The authors declare no conflict of interest. 


\section{REFERENCES}

1. Berson DM, Dunn FA, Takao M. Phototransduction by retinal ganglion cells that set the circadian clock. Science 2002;295:1070-1073.

2. Hattar S, Liao HW, Takao M, Berson DM, Yau KW. Melanopsin-containing retinal ganglion cells: architecture, projections, and intrinsic photosensitivity. Science 2002; 295:1065-1070.

3. Lockley SW, Evans EE, Scheer FA, Brainard GC, Czeisler CA, Aeschbach D. Short-wavelength sensitivity for the direct effects of light on alertness, vigilance, and the waking electroencephalogram in humans. Sleep 2006;29:161-168.

4. Stevens WR. Principles of lighting. London: Constable; 1951.

5. Mills PR, Tomkins SC, Schlangen LJ. The effect of high correlated colour temperature office lighting on employee wellbeing and work performance. J Circadian Rhythms 2007;5:2.

6. Riemersma-van der Lek RF, Swaab DF, Twisk J, Hol EM, Hoogendijk WJ, Van Someren EJ. Effect of bright light and melatonin on cognitive and noncognitive function in elderly residents of group care facilities: a randomized controlled trial. JAMA 2008;299:2642-2655.

7. van Bommel WJM, van den Beld GJ. Lighting for work: a review of visual and biological effects. Light Res Technol 2004:36:255-269.

8. Schulte-Markwort M, Barkmann C, Wessolowski N. Effects of light in schools. In: Proceedings of CIE 2010 "Light Quality and Energy Efficiency"; March 14, 2010; Vienna, Austria. OP30.

9. Deguchi T, Sato M. The effect of color temperature of lighting sources on mental activity level. Ann Physiol Anthropol 1992;11:37-43.

10. Badia P, Myers B, Boecker M, Culpepper J, Harsh JR. Bright light effects on body temperature, alertness, EEG and behavior. Physiol Behav 1991;50:583-588.

11. Hwang MK, An SH, Ban KH, Baek SW. Theory and application of LED light optical design. Part 1. Seoul: Ajin; 2010.

12. Kropotov J. Quantitative EEG, event-related potentials and neurotherapy. San Diego: Academic Press; 2009.

13. Palva S, Palva JM. New vistas for alpha-frequency band oscillations. Trends Neurosci 2007;30:150-158.

14. Figueiro MG, Bierman A, Plitnick B, Rea MS. Preliminary evidence that both blue and red light can induce alertness at night. BMC Neurosci 2009;10:105.

15. Smith MR, Eastman CI. Phase delaying the human circadian clock with blue-enriched polychromatic light. Chronobiol Int 2009;26:709-725.
16. Delorme A, Makeig S. EEGLAB: an open source toolbox for analysis of single-trial EEG dynamics including independent component analysis. J Neurosci Methods 2004;134:921.

17. Kruithof AA. Tubular luminescence lamps for general illumination. Philips Tech Rev 1941;6:65-96.

18. van Bommel WJ. Non-visual biological effect of lighting and the practical meaning for lighting for work. Appl Ergon 2006;37:461-466.

19. Klimesch W, Sauseng P, Hanslmayr S. EEG alpha oscillations: the inhibition-timing hypothesis. Brain Res Rev 2007;53:63-88.

20. Cooper NR, Croft RJ, Dominey SJ, Burgess AP, Gruzelier JH. Paradox lost? Exploring the role of alpha oscillations during externally vs. internally directed attention and the implications for idling and inhibition hypotheses. Int $J$ Psychophysiol 2003;47:65-74.

21. Raven J, Raven JC, Court JH. Manual for Raven's progressive matrices and vocabulary scales. Section 3: Standard progressive matrices (1998 edition). Oxford: Oxford Psychologists Press; 1998.

22. Waltz JA, Knowlton BJ, Holyoak KJ, Boone KB, Mishkin FS, de Menezes Santos M, et al. A system for relational reasoning in human prefrontal cortex. Psychol Sci 1999; 10:119-125.

23. Morsanyi K, Holyoak KJ. Analogical reasoning ability in autistic and typically developing children. Dev Sci 2010; 13:578-587.

24. Adrian ED, Matthews BH. The interpretation of potential waves in the cortex. J Physiol 1934;81:440-471.

25. Avery DH, Kizer D, Bolte MA, Hellekson C. Bright light therapy of subsyndromal seasonal affective disorder in the workplace: morning vs. afternoon exposure. Acta Psychiatr Scand 2001;103:267-274.

26. Hoffmann G, Gufler V, Griesmacher A, Bartenbach C, Canazei M, Staggl S, et al. Effects of variable lighting intensities and colour temperatures on sulphatoxymelatonin and subjective mood in an experimental office workplace. Appl Ergon 2008;39:719-728.

27. Delplanque S, Silvert L, Hot P, Rigoulot S, Sequeira H. Arousal and valence effects on event-related P3a and P3b during emotional categorization. Int J Psychophysiol 2006; 60:315-322.

28. Dolcos F, LaBar KS, Cabeza R. Dissociable effects of arousal and valence on prefrontal activity indexing emotional evaluation and subsequent memory: an event-related fMRI study. Neuroimage 2004;23:64-74.

29. Vandewalle G, Maquet P, Dijk DJ. Light as a modulator of cognitive brain function. Trends Cogn Sci 2009;13:429-438. 